This document is the Accepted Manuscript version of a Published Work that appeared in final form in Industrial \& Engineering Chemistry Research volume 52, number 23, pp. 7854-7866, 2013, copyright (C) American Chemical Society after peer review and technical editing by the publisher. To access the final edited and published work see http://dx.doi.org/10.1021/ie400367m or http://pubs.acs.org/doi/abs/10.1021/ie400367m.

\title{
CENTRALIZED INVERTED DECOUPLING CONTROL
}

\author{
Juan Garrido ${ }^{a *}$, Francisco Vázquez ${ }^{a}$, Fernando Morilla ${ }^{b}$ \\ ${ }^{a}$ Department of Computer Science and Numerical Analysis, University of Cordoba, Campus de \\ Rabanales, 14071, Cordoba, Spain \\ * Fax: (+34)957218729; e-mail: juan.garrido@uco.es \\ ${ }^{\mathrm{b}}$ Department of Computer Science and Automatic Control, UNED, Juan del Rosal 16, 28040, \\ Madrid, Spain
}

\begin{abstract}
:
This paper presents a new methodology of multivariable centralized control based on the structure of inverted decoupling. The method is presented for general $n \times n$ processes, obtaining very simple general expressions for the controller elements with a complexity independent of the system size. The possible configurations and realizability conditions are stated. Then, the specification of performance requirements is carried out from simple open loop transfer functions for three common cases. As a particular case, it is shown that the resulting controller elements have PI structure or filtered derivative action plus a time delay when the process elements are given by first order plus time delay systems. Comparisons with other works demonstrate the effectiveness of this methodology through the use of several simulation examples and an experimental lab process.
\end{abstract}

Keywords: multivariable control, centralized control, decoupling control, inverted decoupling.

\section{Introduction}

Most industrial processes are inherently multivariable. They are made up of several input and output signals, and there are often complicated couplings between them, which may occasion difficulties in feedback controller design. Process control problems are traditionally solved using single-loop PID controllers because they are easily understood and implemented. ${ }^{1}$ These decentralized approaches are adequate when the interactions in different channels of the process 
are modest. ${ }^{2-6}$ However, when interactions are important, the decoupling is often treated inefficiently, e.g., by detuning control loops. Usually, the main loop is tuned to achieve good performance while the other loops are detuned keeping an acceptable interaction with the first loop. In fact, this poor decoupling of multivariable systems is considered by some leading manufactures of controllers as one of the principal control problems in the industry. ${ }^{7}$ In processes with strong interactions, a full matrix controller (centralized control) is advised.

In literature, two approaches of centralized control are usual: a decoupling network combined with a diagonal decentralized controller, or a pure centralized strategy. In the first case, a decoupler $^{8-16}$ is used to minimize interaction or to make the system diagonal dominant; then, the controllers are designed using some decentralized method. Figure 1a shows the general control scheme of this approach where $G(\mathrm{~s}), D(\mathrm{~s})$ and $C(\mathrm{~s})$ are the process matrix, the decoupling matrix and the decentralized control matrix, respectively.

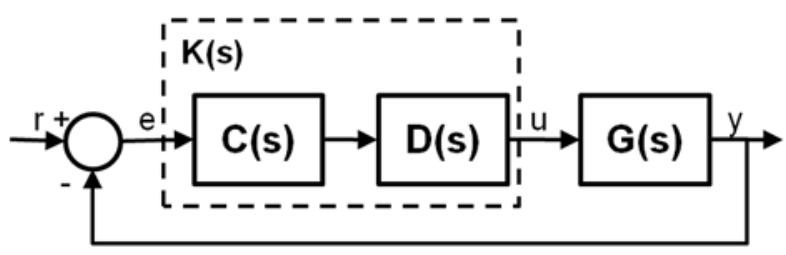

(a)

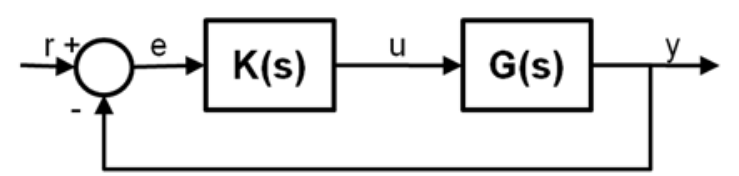

(b)

Figure 1. Centralized control approaches: (a) decoupling control system, (b) purely centralized control system.

On the other hand, Figure 1b represents a pure centralized control system with $K(\mathrm{~s})$ being the n-dimensional full matrix controller. Under the paradigm of decoupling control, some methodologies ${ }^{17-24}$ have been developed using this scheme. Most of them propose to find a $K(\mathrm{~s})$ 
in such a way that the closed loop transfer matrix $H(\mathrm{~s})=G(\mathrm{~s}) \cdot K(\mathrm{~s}) \cdot[I+G(\mathrm{~s}) \cdot K(\mathrm{~s})]^{-1}$ is decoupled over some desired bandwidth. This goal is achieved if the open loop transfer matrix $L(\mathrm{~s})=G(\mathrm{~s}) \cdot K(\mathrm{~s})$ is diagonal over that bandwidth.

The complexity of the resultant controller elements of $K(\mathrm{~s})$ can be very different depending on the methodology. For instance, Wang ${ }^{20}$ obtains a full-dimensional non-PID from a recursive least square optimization problem. $\mathrm{Liu}^{23}$ develops an analytical decoupling control on the basis of the $\mathrm{H}_{2}$ optimal performance specifications. Other methodologies ${ }^{17,21,22}$ reduce the $K(\mathrm{~s})$ elements to PID controllers obtaining as result a multivariable PID control. This reduction is performed because PID controllers are preferred over more advanced controllers in many practical applications (unless PID controls cannot meet the specifications). Although model predictive control (MPC) is becoming the standard solution to multivariable control problems in the process industry, several authors ${ }^{12,20,21}$ assert that MPC is mostly used on a higher level to provide setpoints to the controllers operating with shorter sampling times on the basis level. There can be some difficulties in dealing with the interaction at the MPC level because the bandwidths of the MPC loops are limited. Therefore, the centralized control using multivariable PID controllers or decoupling controllers is an interesting strategy at the process industry.

Most of the previous centralized methodologies use the conventional scheme of Figure 1b in which the process inputs $u$ are derived by a time-weighted combination of the error signals $e$. It has received considerable attention in both control theory and applications for several years. In this case, specifying a desired diagonal matrix $L(\mathrm{~s})$ or $H(\mathrm{~s})$ as requirement in the design, the matrix $K(\mathrm{~s})$ can be calculated according to (1) or (2), respectively.

$$
\begin{aligned}
& K(s)=G^{-1}(s) \cdot L(s) \\
& K(s)=G^{-1}(s) \cdot\left(H^{-1}(s)-I\right)^{-1}
\end{aligned}
$$


Nevertheless, the requirements of properness and causality for controller implementation can make this direct calculation difficult, especially for systems with high dimension or time delays. Consequently, these methods are likely to need a burdensome numerical computation effort or, instead of it, some kind of approximation or reduction to simplify the calculus, even for $2 \times 2$ systems. For instance, in process with time delays, the final controller elements could be complex non-rational transfer functions that should be finally approximated before implementation. In addition, when the size of the system increases, the complexity of the controller elements and calculations is increased as well.

This work proposes a new centralized control methodology which is based on the idea of extrapolate the structured of inverted decoupling networks ${ }^{15,25}$ to the centralized control scheme. The new scheme is called centralized inverted decoupling control and it derives a process input as a time-weighted combination of one error signal and other time-weighted process inputs. As a consequence of this structure, it is possible to achieve easily the desired requirements without any approximation and using very simple controller elements in $K(\mathrm{~s})$. In addition, in contrast to the conventional centralized scheme, the complexity of the transfer functions of the controller elements is independent of the system size, as it will be demonstrated.

An initial version of this methodology was introduced only for $2 \times 2$ processes. ${ }^{26,}{ }^{27}$ In this work, further research was performed focusing on stable process with possible right half plane (RHP) zeros and time delays. Additionally, the formulation of this new centralized control scheme is generalized to $n \times n$ processes. The paper is structured as follows. Section 2 presents this generalization of centralized inverted decoupling control for $n \times n$ systems and the different possible configurations. Furthermore, the $2 \times 2$ case is described in more detail and some expressions for $3 \times 3$ processes are given. In Section 3 , the aspects related to realizability conditions and performance specifications are discussed. Section 4 is focused on the structure of 
the final controller elements, its approximation to PID structure, and the particular case of processes in which all elements are first order plus time delay (FOPDT) systems. In Section 5, the performance of the proposed method is tested and compared with other techniques using several simulation examples and a real quadruple tank process. Finally, conclusions are summarized in Section 6.

\section{Centralized inverted decoupling control for $n \times n$ processes}

In order to develop the centralized inverted decoupling control for a square process $G(\mathrm{~s})$ with $n$ inputs and $n$ outputs, a matrix representation of $K(\mathrm{~s})$ is proposed as shown in Figure 2. The matrix $K(\mathrm{~s})$ is split into two blocks: a matrix $K d(\mathrm{~s})$ in the direct path (between error signals $e$ and process inputs $u$ ) and a matrix $K o(\mathrm{~s})$ in a feedback loop (between process inputs $u$ and error signals $e$ ).

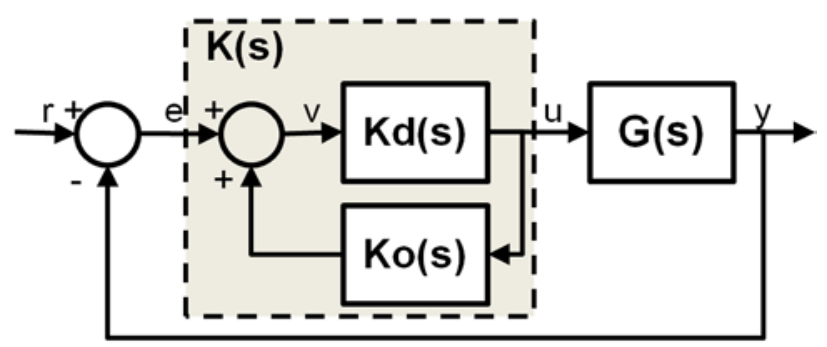

Figure 2. Centralized inverted decoupling control scheme.

According to the inverted decoupling structure, in $K d(\mathrm{~s})$ there must be only $n$ elements (the rest of elements must be zero) which try to directly connect the error signals with the process inputs $u$. $K o(\mathrm{~s})$ feedback the process inputs $u$ toward the controller inputs in order to decouple the system. In contrast to $K d(\mathrm{~s}), K o(\mathrm{~s})$ must have only $n$ zero elements, which correspond with the transpose non-zero elements of $K d(\mathrm{~s})$ since the signal flow direction in $K o(\mathrm{~s})$ is opposite that of $K d(\mathrm{~s})$ and the relationships in $K d(\mathrm{~s})$ are not required in the matrix $K o(\mathrm{~s})$. For instance, in a 
$3 \times 3$ system, if element $K d(2,3)$ is specified as a direct connection between $u_{2}$ and $e_{3}$, there will not be feedback from $u_{2}$ toward $e_{3}$, and therefore, the element $K o(3,2)$ must be zero.

From the controller representation given in Figure 2, the expression of the whole controller matrix $K(\mathrm{~s})$ is obtained as follows:

$$
K(s)=K d(s) \cdot(I-K o(s) \cdot K d(s))^{-1} .
$$

If equations (1) and (3) are combined, the expression that relates the conventional centralized design to the $K d(\mathrm{~s})$ and $K o(\mathrm{~s})$ matrices of centralized inverted structure can be found. However, as it a complex expression, it is easier to work with its inverse, which is very simple, as follows:

$$
K d^{-1}(s)-K o(s)=L^{-1}(s) \cdot G(s)
$$

The controller elements of the two matrices, $K d(\mathrm{~s})$ and $K o(\mathrm{~s})$, can be calculated using this last expression. Assuming that the open loop transfer matrix $L(\mathrm{~s})$ must be diagonal to achieve a decoupled closed loop response, the main advantage of equation (4) is its simplicity, regardless of the system size, because the matrix $L(\mathrm{~s})$ is specified to be diagonal and the resulting subtraction of the inverse of $K d(\mathrm{~s})$ and $K o(\mathrm{~s})$ is a transfer matrix with only one element to be calculated in each position.

Note that $K d(\mathrm{~s})$ has to be non-singular because it is inverted, and therefore, when its elements are selected, only one element in each row and column can be chosen. As a result, for an $n \times n$ process there are $n$ ! possible configurations of $K d(\mathrm{~s})$. To name these possibilities, the authors propose a notation in which the indicated number corresponds to the column with the selected element. For instance, in a $3 \times 3$ system, configuration 1-2-3 means that elements $K d(1,1), K d(2,2)$ and $K d(3,3)$ are selected; configuration 2-3-1 means that elements $K d(1,2), K d(2,3)$ and $K d(3,1)$ are chosen, and so on. The expression of the controller elements for each configuration is different, which is interesting because some choices can result in non-realizable controller 
elements. Therefore, the configuration can be selected depending on the realizability, which will be discussed later.

From (4) it is possible to obtain the general expressions of the centralized inverted decoupling control for $n \times n$ processes. If the configuration $p_{1}-p_{2}-\ldots-p_{i}-\ldots-p_{n-1}-p_{n}$ is chosen, the non-zero elements of the $K d(\mathrm{~s})$ and $K o(\mathrm{~s})$ matrices are given by (5) and (6), respectively. The transfer functions of the desired open loop processes $l_{i}(\mathrm{~s})$ can be specified in any way that assures the realizability of these controller elements. In addition, it is usually preferable to chose simple transfer functions $l_{i}(\mathrm{~s})$ in such a way that the performance requirements can be specified easily.

$$
\begin{aligned}
& k d_{i j}(s)=\frac{l_{j}(s)}{g_{j i}(s)} \quad \forall i ; \quad j=p_{i} \\
& k o_{i j}(s)=\frac{-g_{i j}(s)}{l_{i}(s)} \quad \forall i, j ; / i \neq p_{j}
\end{aligned}
$$

From these general expressions, the following advantages over conventional centralized control are concluded:

- Controller elements do not contain sum of transfer functions, and therefore they are easy to design. In some multivariable processes, even if the elements of the system have simple dynamics, conventional centralized control may result in complicated controller elements difficult to implement, as follows:

$$
\frac{2.3}{(0.3 s+1)(0.5 s+1)} e^{-5 s}-\frac{1}{(6 s+1)(s+1)} e^{-2 s} .
$$

- The complexity of the controller elements and open loop processes is always the same, independent of the system size. With conventional centralized control, these elements become more complex as the size of the process increases.

Nonetheless, the proposed method presents the same important disadvantage as inverted decoupling: it cannot be applied to processes with RHP zeros in its determinant. To achieve 
internal stability, these multivariable RHP zeros should be included into the open loop processes. In the conventional centralized control structure, these zeros can be specified in the desired transfer functions $l_{i}(\mathrm{~s})$. However, this is not possible using centralized inverted decoupling control because such RHP zeros would appear as unstable poles in some controller elements $k o_{i j}(\mathrm{~s})$. Although the single controller elements were stable, if the multivariable RHP zeros are not specified in the open loop processes, the whole control structure $(K)$ would be unstable since these zeros would be internally included as unstable poles in the inverse of $G(\mathrm{~s})$, as follows:

$$
K(s)=\left(K d^{-1}(s)-K o(s)\right)^{-1}=G^{-1}(s) \cdot L(s) .
$$

A special case arises when the multivariable RHP zero is associated with a single output and is therefore included in the process transfer functions of the same row. Then, centralized inverted decoupling control can be applied because the RHP zero will be cancelled.

\subsection{Centralized inverted decoupling control for $2 \times 2$ processes}

Next, a detailed study of the proposed methodology for $2 \times 2$ processes is performed using expressions (5) and (6). In this case ( $n=2)$, there are two possible configurations to select for the non-zero elements of $K d(\mathrm{~s})$ : diagonal elements (configuration 1-2) or off-diagonal elements (configuration 2-1).

Using configuration 1-2 (depicted in Figure 3), the following expressions (9) for the non-zero controller elements are obtained from (5) and (6). The elements $l_{1}(\mathrm{~s})$ and $l_{2}(\mathrm{~s})$ are the desired open loop transfer functions.

$$
\begin{array}{ll}
k d_{11}(s)=\frac{l_{1}(s)}{g_{11}(s)} & k o_{12}(s)=\frac{-g_{12}(s)}{l_{1}(s)} \\
k o_{21}(s)=\frac{-g_{21}(s)}{l_{2}(s)} & k d_{22}(s)=\frac{l_{2}(s)}{g_{22}(s)}
\end{array}
$$




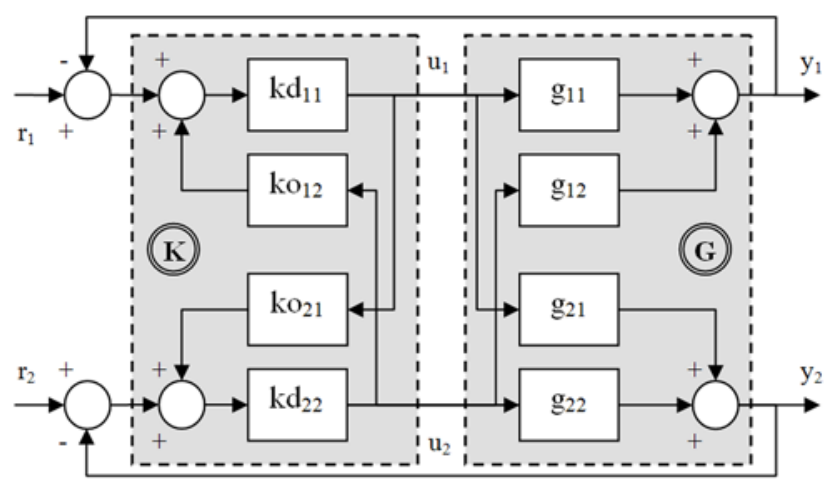

Figure 3. Centralized inverted decoupling control for $2 \times 2$ processes (configuration 1-2)

In the configuration 2-1 (Figure 4), the general expressions for the controller elements are given by (10), which are obtained from (5) and (6).

$$
\begin{array}{ll}
k o_{11}(s)=\frac{-g_{11}(s)}{l_{1}(s)} & k d_{12}(s)=\frac{l_{2}(s)}{g_{21}(s)} \\
k d_{21}(s)=\frac{l_{1}(s)}{g_{12}(s)} & k o_{22}(s)=\frac{-g_{22}(s)}{l_{2}(s)}
\end{array}
$$

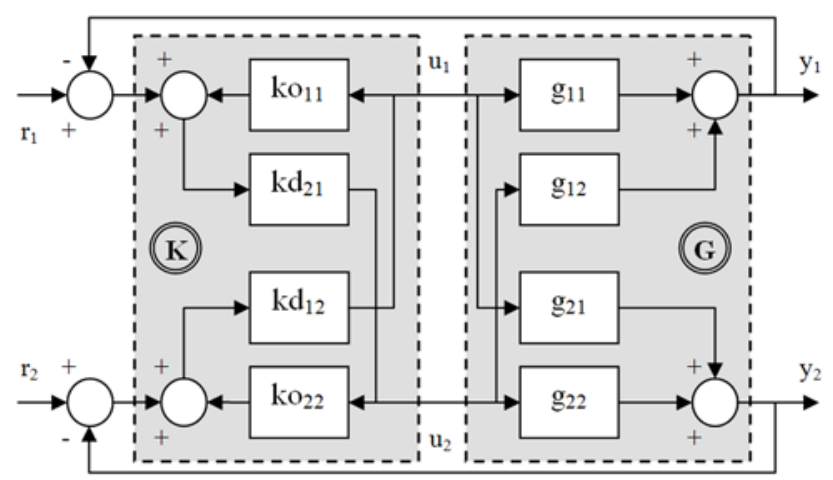

Figure 4. Centralized inverted decoupling control for $2 \times 2$ processes (configuration 2-1)

The corresponding general expressions for conventional centralized decoupling control for $2 \times 2$ processes that are obtained according to (1) are given by (11). It is easy to find that the expressions of the proposed method in both (9) and (10) are much simpler than those of the conventional centralized control elements in (11). 


$$
K(s)=\left(\begin{array}{ll}
k_{11}(s) & k_{12}(s) \\
k_{21}(s) & k_{22}(s)
\end{array}\right)=\frac{\left(\begin{array}{ll}
g_{22}(s) l_{1}(s) & -g_{12}(s) l_{2}(s) \\
-g_{21}(s) l_{1}(s) & g_{11}(s) l_{2}(s)
\end{array}\right)}{g_{11}(s) g_{22}(s)-g_{12}(s) g_{21}(s)}
$$

\subsection{Centralized inverted decoupling control for $3 \times 3$ processes}

The procedure is similar for $3 \times 3$ processes, although in this case there are six possible configurations according to the three elements of $K d(\mathrm{~s})$ selected to be non-zero. For instance, using configuration 1-2-3 in (4), the equation (12) is achieved, and from it, the expressions for controller elements given in (13) are easily obtained, which are equivalent to those obtained using (5) and (6). The expressions are as simple as in $2 \times 2$ systems.

$$
\begin{aligned}
& \left(\begin{array}{ccc}
\frac{1}{\mathrm{kd}_{11}(s)} & -\mathrm{ko}_{12}(s) & -\mathrm{ko}_{13}(s) \\
-\mathrm{ko}_{21}(s) & \frac{1}{\mathrm{kd}_{22}(s)} & -\mathrm{ko}_{23}(s) \\
-\mathrm{ko}_{31}(s) & -\mathrm{ko}_{32}(s) & \frac{1}{\mathrm{kd}_{33}(s)}
\end{array}\right)=\left(\begin{array}{ccc}
\frac{\mathrm{g}_{11}(s)}{l_{1}(s)} & \frac{\mathrm{g}_{12}(s)}{l_{1}(s)} & \frac{\mathrm{g}_{13}(s)}{l_{1}(s)} \\
\frac{\mathrm{g}_{21}(s)}{l_{2}(s)} & \frac{\mathrm{g}_{22}(s)}{l_{2}(s)} & \frac{\mathrm{g}_{23}(s)}{l_{2}(s)} \\
\frac{\mathrm{g}_{31}(s)}{l_{3}(s)} & \frac{\mathrm{g}_{32}(s)}{l_{3}(s)} & \frac{\mathrm{g}_{33}(s)}{l_{3}(s)}
\end{array}\right) \\
& \mathrm{kd}_{11}(s)=\frac{\mathrm{l}_{1}(\mathrm{~s})}{\mathrm{g}_{11}(\mathrm{~s})} \quad k \mathrm{o}_{12}(\mathrm{~s})=\frac{-\mathrm{g}_{12}(\mathrm{~s})}{\mathrm{l}_{1}(\mathrm{~s})} \quad k \mathrm{o}_{13}(\mathrm{~s})=\frac{-\mathrm{g}_{13}(\mathrm{~s})}{\mathrm{l}_{1}(\mathrm{~s})} \\
& \mathrm{ko}_{21}(s)=\frac{-\mathrm{g}_{21}(s)}{l_{2}(s)} \quad k \mathrm{~d}_{22}(s)=\frac{l_{2}(s)}{\mathrm{g}_{22}(s)} \quad k \mathrm{o}_{23}(s)=\frac{-\mathrm{g}_{23}(s)}{l_{2}(s)} \\
& \mathrm{ko}_{31}(s)=\frac{-\mathrm{g}_{31}(s)}{l_{3}(s)} \quad k \mathrm{o}_{32}(s)=\frac{-\mathrm{g}_{32}(s)}{l_{3}(s)} \quad k \mathrm{~d}_{33}(s)=\frac{\mathrm{l}_{3}(s)}{\mathrm{g}_{33}(s)}
\end{aligned}
$$

\section{Design considerations}

\subsection{Realizability}

The realizability requirement for the controller is that all of its elements must be proper, causal and stable. For systems with time delays or non-minimum phase zeros, direct calculations can lead to elements with prediction or RHP poles. In the proposed methodology, there are two issues regarding controller realizability that have to be studied: firstly, it is necessary to check whether it is possible to achieve realizability using the chosen configuration; and secondly, after confirming the previous condition, it is necessary to determine how to specify the desired open 
loop transfer functions $l_{i}(\mathrm{~s})$. In this section, the conditions that a specified configuration needs to satisfy in order to be realizable are stated. Furthermore, the constraints on the open loop processes to obtain such realizability are indicated as well.

In the controller expressions (5) and (6), each desired open loop transfer function $l_{i}(\mathrm{~s})$ appears associated to the process transfer functions $g_{i j}(\mathrm{~s})$ of the same row $i$. Therefore, there are three aspects to take into consideration and to be inspected for each row of the process matrix $G(\mathrm{~s})$ :

- Non-causal time delays must be avoided in controller elements. If $g_{i k}(\mathrm{~s})$ is the transfer function of the row $i$ with the smallest time delay $\theta_{i k}$, the element $k d_{i k}(\mathrm{~s})$ of $K d(\mathrm{~s})$ should be selected to be in the direct path between the error signals and the process inputs (it should be different from zero). In addition, the time delay $\theta_{i}$ of the open loop transfer function $l_{i}(\mathrm{~s})$ must fulfill:

$$
\theta_{i k} \leq \theta_{i} \leq \min _{j \neq k}\left(\theta_{i j}\right)
$$

where $\theta_{i j}$ is the time delay of $g_{i j}(\mathrm{~s})$.

- Controller elements must be proper, that is, the relative degrees must be greater or equal than zero. Similarly to the previous case, if $g_{i k}(\mathrm{~s})$ is the transfer function of the row $i$ with the smallest relative degree $r_{i k}$, the element $k d_{k i}(\mathrm{~s})$ should be different from zero. In addition, the relative degree $r_{i}$ of the open loop process $l_{i}(\mathrm{~s})$ must fulfill:

$$
r_{i k} \leq r_{i} \leq \min _{j \neq k}\left(r_{i j}\right) .
$$

- When some transfer function $g_{i m}(\mathrm{~s})$ has a RHP zero, the element $k d_{m i}(\mathrm{~s})$ of $K d(\mathrm{~s})$ should not be selected in the direct path, in order to avoid this zero becomes a RHP pole in some controller element. When the zero appears in all elements of the same row, it is necessary to check its multiplicity in each element of the row. Again, if $g_{i k}(\mathrm{~s})$ is the process transfer function of the row $i$ with the smallest RHP zero multiplicity $\eta_{i k}$, the element $k d_{k i}(\mathrm{~s})$ should be chosen to be in the 
direct path (it should be non-zero). This RHP zero must appear in the open loop process $l_{i}(\mathrm{~s})$ with a multiplicity $\eta_{i}$ that fulfills (16). This condition must be fulfilled for each different multivariable RHP zero $z_{x}$.

$$
\eta_{i k} \leq \eta_{i} \leq \min _{j \neq k}\left(\eta_{i j}\right)
$$

From (14), (15) and (16), it can be deduced that there are more possibilities for selecting a realizable configuration when the smallest value (time delay, relative degree or RHP zero multiplicity) is shared by two or more transfer functions of the same row. However, in that case, the flexibility to specify this value (time delay, relative degree of RHP zero multiplicity) in the desired open loop process of that row is limited to this common smallest value.

When two or more elements of $K d(\mathrm{~s})$ must be selected necessarily in the same column to satisfy the previous conditions in all rows, there are no realizable configurations. Then, it is necessary to insert an additional diagonal block $N(\mathrm{~s})$ between the process $G(\mathrm{~s})$ and the controller in order to modify the system trying to force the non-realizable elements into realizability. Then, the method is applied to the new process $G^{N}(\mathrm{~s})=G(\mathrm{~s}) \cdot N(\mathrm{~s})$.

$N(\mathrm{~s})$ is a diagonal transfer matrix with the necessary extra dynamics. If there are no realizability problems in the row $i$, the $n_{i i}(\mathrm{~s})$ element is equal to the unity. If the non-realizability comes from an element with a non-causal time delay, an additional time delay $\theta_{i i}$ is specified in $n_{i i}(\mathrm{~s})$. If it comes from a RHP zero $z$, which becomes into unstable poles, this RHP zero is added together with its mirrored pole and the proper multiplicity $\eta_{i i}$ as in (17). If it comes from a properness problem, a simple stable pole with a small time constant $\tau$ and the adequate multiplicity $r_{i i}$ is inserted as it is shown in (18).

$$
\left(\frac{-s+z}{s+Z}\right)^{\eta_{i i}}
$$




$$
\frac{1}{(\tau s+1)^{r_{i i}}}
$$

Generally, it is preferable to add the minimum extra dynamics. Therefore, after checking the necessary additional dynamics of each configuration, it is chosen that one with fewer RHP zeros or time delays in $N(\mathrm{~s})$. More detailed information about this issue is given in other works. ${ }^{9}$

\subsection{Performance specifications}

After assuring that it is possible to achieve realizability with the chosen configuration, the $n$ desired open loop processes $l_{i}(\mathrm{~s})$ must be specified. Two aspects must be taken into account in order to define each transfer function $l_{i}(\mathrm{~s})$ : the realizability of the controller elements and the performance specifications of the corresponding closed loop transfer function $h_{i}(\mathrm{~s})$. Since the closed loop response must be stable and without steady state errors due to setpoint or load changes, the open loop transfer function $l_{i}(\mathrm{~s})$ must contain an integrator. Then, the following general expression (19) is proposed.

$$
l_{i}(\mathrm{~s})=\frac{k_{i} \cdot \bar{l}_{i}(\mathrm{~s})}{\mathrm{s}}
$$

The parameter $k_{i}$ becomes a tuning parameter to meet design specifications. The transfer function $\bar{l}_{i}$ (s) takes into account the realizability conditions from (14) to (16) and consequently, its general expression is given by (20). Regarding condition (15) it is important to note that the integrator in (19) already provides an extra relative degree to $l_{i}(\mathrm{~s})$.

$$
\bar{l}_{i}(\mathrm{~s})=e^{-\theta_{i} s} \cdot \frac{1}{(\tau s+1)^{r_{i}}} \cdot \prod_{x=1}^{N z}\left(\frac{-s+z_{x}}{s+z_{x}}\right)^{\eta x_{i}}
$$

Next, it is explained how to specify the different parameters of the open loop transfer functions when attention is addressed to some of the three simple cases listed in Table 1. Many other cases can be studied; however, the three cases shown in Table 1 arise very often and direct tuning expressions can be obtained from them. Firstly, it is preferable to specify simple open loop transfer 
functions so that their parameters can be easily tuned to meet the desired specifications. It also contributes to achieve simpler controller elements. As many industrial processes can be modelled by first or second order systems, the relative degrees of the process elements of the transfer matrix $G(\mathrm{~s})$ are usually one or two. Therefore, it is very common that the $l_{i}(\mathrm{~s})$ transfer functions need to be specified with relative degree one or two in order to fulfil condition (15), as in the cases of Table 1 . Secondly, some time delay can be necessary in $l_{i}(\mathrm{~s})$ to fulfil condition (14). Although condition (16) has been developed in section 3.1, no case with RHP zeros has been included in Table 1 because it is not very usual to find open loop processes with RHP zeros. The third case is somewhat special because it is dedicated to processes that show an integrator associated to one output.

Table 1. Three simple cases to define the open loop transfer function.

\begin{tabular}{ccc}
\hline Case & $\overline{l_{i}}(\mathrm{~s})$ & $l_{i}(\mathrm{~s})$ \\
\hline 1 & $e^{-\theta_{i} s}$ & $\frac{k_{i}}{s} \cdot e^{-\theta_{i} s}$ \\
2 & $\frac{1}{\lambda s+1} \cdot e^{-\theta_{i} s}$ & $\frac{k_{i}}{s \cdot(\lambda \cdot s+1)} \cdot e^{-\theta_{i} s}$ \\
3 & $\frac{\left(s+z_{i}\right)}{s} \cdot e^{-\theta_{i} s}$ & $\frac{k_{i} \cdot\left(s+z_{i}\right)}{s^{2}} \cdot e^{-\theta_{i} s}$ \\
\hline
\end{tabular}

\subsubsection{Case 1}

In this case, it is necessary at most a time delay to achieve realizability. The corresponding expressions for $\bar{l}_{i}(\mathrm{~s})$ and $l_{i}(\mathrm{~s})$ are given in the first row of Table 1 . The imposition of relative stability specifications is enough to guarantee the stability of the closed loop transfer function $h_{i}(\mathrm{~s})=l_{i}(\mathrm{~s}) /\left(1+l_{i}(\mathrm{~s})\right)$. It can be found that the phase margin $\varphi_{m}$ and gain margin $A_{m}$ of $l_{i}(\mathrm{~s})$ are given by (21) and (22), respectively, at the corresponding frequencies $\omega_{c p}$ and $\omega_{c g}$ shown in (21) and (22) as well. 


$$
\begin{aligned}
& \varphi_{m}=90-\frac{180 k_{i} \theta_{i}}{\pi} \quad \omega_{c p}=k_{i} \\
& A_{m}=\frac{\pi}{2 k_{i} \theta_{i}} \quad \omega_{c g}=\frac{\pi}{2 \theta_{i}}(22)
\end{aligned}
$$

Both margins are related as follows:

$$
\varphi_{m}=90-\frac{20}{A_{m}} .(23)
$$

If a phase margin less than $90^{\circ}$ or a gain margin greater than 1 is specified, the value of $k_{i}$ can be directly calculated by means of (24) or (25), respectively. Increasing $k_{i}$ makes the closed loop response faster. However, it implies smaller values of phase margin and gain margin and consequently, the control system robustness is reduced.

$$
\begin{aligned}
& k_{i}=\frac{\pi\left(90^{\circ}-\varphi_{m}\right)}{180^{\circ} \cdot \theta_{i}} \\
& k_{i}=\frac{\pi}{2 A_{m} \theta_{i}}
\end{aligned}
$$

If $\overline{l_{i}}(\mathrm{~s})$ do not have time delay $\left(\theta_{i}=0\right)$ so it is equal to the unity, the open loop function $l_{i}(\mathrm{~s})=$ $k_{i} / \mathrm{s}$ has a phase margin of $90^{\circ}$ and an infinite gain margin independently of the $k_{i}$ parameter. In addition, the closed loop transfer function $h_{i}(\mathrm{~s})$ is a traditional first order system with time constant $T_{i}=1 / k_{i}$, as follows:

$$
h_{i}(\mathrm{~s})=\frac{k_{i} / s}{k_{i} / s+1}=\frac{1}{T_{i} s+1} .
$$

Therefore, the desired closed loop time constant $T_{i}$ is proposed as specification to determine $k_{i}$ instead of relative stability margins. This situation is equivalent to one of the most common cases in the methodologies of IMC $\operatorname{control}^{28}$ or affine parameterization. ${ }^{29}$

\subsubsection{Case 2}


This case arises when the lowest relative degree of the process elements $g_{i j}(\mathrm{~s})$ of the row $i$ associated to $l_{i}(\mathrm{~s})$ is two. In order to obtain proper controller elements, it is necessary to include an extra pole in $\overline{l_{i}}(\mathrm{~s})$. The second row of Table 1 shows the corresponding expressions for $\overline{l_{i}}(\mathrm{~s})$ and $l_{i}(\mathrm{~s})$. The gain margin of this transfer function $l_{i}(\mathrm{~s})$ is given by $(27)$ at the frequency $\omega_{c g}$ that fulfills the condition (28). In this work, it is proposed to calculate $\lambda$ from (28) after specified the frequency $\omega_{c g}$. Then, $k_{i}$ is obtained from (27) after choosing a desired gain margin $A_{m}$.

$$
\begin{aligned}
& A_{m}=\frac{1}{k_{i} \cdot \lambda \cdot \tan \left(\omega_{c g} \theta_{i}\right) \cdot \sin \left(\omega_{c g} \theta_{i}\right)} \\
& \tan \left(\omega_{c g} \theta_{i}\right)=\frac{1}{\lambda \cdot \omega_{c g}}
\end{aligned}
$$

If there are no time delays in $l_{i}(\mathrm{~s})\left(\theta_{i}=0\right)$, the gain margin specification can be replaced by time response specifications because the corresponding closed loop transfer function $h_{i}(\mathrm{~s})$ is given by a second order system as follows:

$$
h_{i}(\mathrm{~s})=\frac{\frac{k_{i}}{s(\lambda \cdot s+1)}}{1+\frac{k_{i}}{s(\lambda \cdot s+1)}}=\frac{\frac{k_{i}}{\lambda}}{s^{2}+\frac{1}{\lambda} s+\frac{k_{i}}{\lambda}} .
$$

The poles of $h_{i}(\mathrm{~s})$ in (29) are characterized by the undamped natural frequency $\omega_{n}$ and the damping factor $\xi$ given by (30).

$$
\omega_{n}=\sqrt{k_{i} / \lambda} \quad \xi=\frac{1}{2 \sqrt{k_{i} \cdot \lambda}}
$$

\subsubsection{Case 3}

This special case appears when all elements $g_{i j}(\mathrm{~s})$ of the row $i$ consist of an integrator, that should be specified in $\overline{l_{i}}(\mathrm{~s})$ in order to maintain the integral action in the elements of $K d(\mathrm{~s})$. Otherwise, the integrator would be cancelled in the corresponding $K d(\mathrm{~s})$ element according to (5) and consequently, zero error in steady state would not be guaranteed in that loop. Because of this 
additional integrator, the relative degree of $l_{i}(\mathrm{~s})$ increases one unit. If no additional degrees are needed, an extra zero $z_{i}$ can be included to keep the proper relative degree in $l_{i}(\mathrm{~s})$ and fulfill condition (15). Then, the corresponding expressions for $\bar{l}_{i}(\mathrm{~s})$ and $l_{i}(\mathrm{~s})$ are given by the third row of Table 1 . In these conditions, the gain margin of $l_{i}(\mathrm{~s})$ is given by (31) at the frequency $\omega_{c g}$ that fulfills condition (32). Authors propose to fix the value of the zero $z_{i}$, subsequently, to calculate the frequency $\omega_{c g}$ from (32) and finally, to obtain the parameter $k_{i}$ from (31).

$$
\begin{aligned}
& A_{m}=\frac{\omega_{c g}^{2}}{k_{i} \sqrt{\omega_{c g}^{2}+z_{i}^{2}}} \\
& \arctan \left(\frac{\omega_{c g}}{z_{i}}\right)-\omega_{c g} k_{i}=0
\end{aligned}
$$

If $\overline{l_{i}}(\mathrm{~s})$ do not contain time delays, the gain margin specification can be replaced again by time response specifications since the closed loop transfer function is given by a second order transfer function with a zero at $s=-z_{i}$, as shown in (33). Its poles are characterized by the natural frequency $\omega_{n}$ and the damping factor $\xi$ given by (34). Therefore, fixing the value of the zero $z_{i}$, it is possible to modify the values of $\omega_{n}$ and $\xi$ through the parameter $k_{i}$. As a particular case, adjusting $k_{i}=4 \cdot z_{i}$, a critical damping response $(\xi=1)$ is obtained with $\omega_{n}=2 \cdot z_{i}$.

$$
\begin{aligned}
& h_{i}(s)=\frac{\frac{k_{i}\left(s+z_{i}\right)}{s^{2}}}{1+\frac{k_{i}\left(s+z_{i}\right)}{s^{2}}}=\frac{k_{i}\left(s+z_{i}\right)}{s^{2}+k_{i} s+k_{i} z_{i}} \\
& \omega_{n}=\sqrt{k_{i} z_{i}} \quad \xi=\sqrt{\frac{k_{i}}{4 z_{i}}}
\end{aligned}
$$

In the three cases explained, the parameter $k_{i}$ acts as a degree of freedom to modify the corresponding closed loop performance and to achieve new specifications almost independently of the other loops. 


\section{Multivariable PID control by inverted decoupling}

Replacing open loop process $l_{i}(\mathrm{~s})$ in (5) and (6) with the general expression (19) and using the configuration $p_{1}-p_{2}-\ldots-p_{i}-\ldots-p_{n-1}-p_{n}$, the following expressions (35) and (36) are obtained for the elements of the centralized inverted decoupling control:

$$
\begin{aligned}
& k d_{i j}(s)=k_{j} \frac{\bar{l}_{o j}(s)}{g_{o j i}(s)} \frac{1}{s} e^{-s\left(\theta_{j}-\theta_{j i}\right)} \quad \forall i ; \quad j=p_{i}, \\
& k o_{i j}(s)=-\frac{g_{o i j}(s)}{k_{i} \cdot \bar{l}_{o i}(s)} \cdot s \cdot e^{-s\left(\theta_{i j}-\theta_{i}\right)} \quad \forall i, j ; / i \neq p_{j} .
\end{aligned}
$$

where $g_{o i j}(\mathrm{~s})$ is the delay free part of $g_{i j}(\mathrm{~s})$ and $\bar{l}_{o i}(s)$ is the delay free part of $\overline{l_{i}}(\mathrm{~s})$ after extracting its corresponding time delays $\theta_{i j}$ and $\theta_{i}$, respectively. From (35), it can be concluded that the non-zero elements of $K d(\mathrm{~s})$ have integral action, and consequently, they might be approximated to PID structure. On the other hand, the non-zero elements of $K o(\mathrm{~s})$ are compensators with derivative action plus time delay and certain dynamics associated to the corresponding process element $g_{i j}(\mathrm{~s})$. Note that care should be taken with the derivative action. In order to avoid high frequency noise amplification, it is necessary to assure that this derivative action is filtered enough in each controller element $k o_{i j}(\mathrm{~s})$.

\subsection{Approximation of controller elements}

The controller elements obtained from (35) and (36) are rational transfer function plus a possible time delay. However, if the dynamics of some process element $g_{i j}(\mathrm{~s})$ or desired open loop $l_{i}(\mathrm{~s})$ are too complex, some controller elements can result in a high order and their approximation can be advisable for implementation. This approximation can be carried out using different techniques like parametric approximation in the frequency domain based on least squares estimators ${ }^{30}$ or prediction error methods; or model reduction techniques based on balanced residualtization. ${ }^{31}$ 
As mentioned previously, the non-zero elements of $K d(\mathrm{~s})$ can be approximated to PID structure. In this work, the parallel form shown in (37) is used, where $K_{P}$ is the proportional constant, $K_{I}$ the integral constant, $K_{D}$ the derivative constant and $T_{F}$ is the derivative filter constant. Although the parameters have little physical interpretation in this form, it is the most flexible structure that allows independency between the different control actions.

$$
k^{P I D}(\mathrm{~s})=K_{P}+\frac{K_{I}}{s}+\frac{K_{D} s}{T_{F} s+1}
$$

In this work, a parametric approximation in the frequency domain is proposed for the PID reduction. Instead of reducing directly the controller element, the authors propose to remove the integrator of the controller element $k d_{i j}(\mathrm{~s})$ and apply the model reduction to the inverse of this result $m(\mathrm{~s})$. The new stationary gain $k_{0}$, as shown in (38), would be identified with the integral constant $K_{I}$.

$$
k_{0}=\lim _{s \rightarrow 0}[k d(\mathrm{~s}) \cdot \mathrm{s}]=\lim _{s \rightarrow 0}[m(\mathrm{~s})]
$$

Without the integrator and after dividing by $k_{0}$, the frequency response of the inverse of $m(s)$ should be approximated according to (39). The resultant approximation must be identified with the inverse of (37) multiplied by $K_{I} / \mathrm{s}$.

$$
\frac{k_{0}}{m(\mathrm{~s})} \approx \frac{b_{1} s+1}{a_{2} s^{2}+a_{1} s+1}=\frac{T_{F} s+1}{\left(\frac{K_{P} T_{F}+K_{D}}{K_{I}}\right) s^{2}+\left(\frac{K_{P}}{K_{I}}+T_{F}\right) s+1} .
$$

In this way, the PID gains after approximation can be identified as follows

$$
\begin{aligned}
& K_{P}=\left(a_{1}-b_{1}\right) \cdot k_{0} \\
& K_{I}=k_{0} \\
& K_{D}=a_{2} \cdot k_{0}-b_{1} \cdot\left(a_{1}-b_{1}\right) \cdot k_{0} \\
& T_{F}=b_{1}
\end{aligned} .
$$

For PI approximation, $K_{D}$ and $T_{F}$ are removed; therefore, the coefficients $b_{1}$ and $a_{2}$ would be zero. Both PI and PID approximations are obtained, the one with the best fit is chosen. 
In a similar way, the $K o(s)$ elements in (36) can be approximated to the structure shown in (41), which consists of a filtered derivative action plus a possible time delay. Before reduction, it is suggested to remove the time delay and the zero in $s=0$ and apply the approximation to (42). In this way, it is obtained that $K_{D}=b_{0} / a_{0}$ and $T_{F}=a_{1} / a_{0}$.

$$
\begin{aligned}
& k^{D}(s)=s\left(\frac{K_{D}}{T_{F} s+1}\right) \cdot e^{-s \theta} \\
& m(s) \cong \frac{b_{0}}{a_{1} s+a_{0}}
\end{aligned}
$$

\subsection{Application to FOPTD systems}

Most industrial processes are open loop stable without oscillatory response for unit step inputs. Therefore, high order processes are often simplified to FOPDT systems before the control system design. In this section and as a particular case, the proposed methodology is applied to multivariable systems in which all of its elements are modelled or simplified by FOPDT processes. Therefore, the process elements $g_{i j}(\mathrm{~s})$ are given by a transfer function as follows:

$$
g_{i j}(s)=\frac{k_{i j}}{T_{i j} s+1} e^{-\theta_{j i} s} .
$$

Assuming a realizable configuration and since all elements are stable, without RHP zeros and with the same relative degree equal to the unity, the specification of $l_{i}(\mathrm{~s})$ can be done according to the case 1 of Table 1 , because time delays are the only aspect to take into account according to (14) in order to achieve realizability.

In order to avoid time delays in the element $k d_{i j}(\mathrm{~s})$, the time delay of $l_{i}(\mathrm{~s})$ is set to the time delay of the associated process element $g_{j i}(\mathrm{~s})$ in (35). In this way, the controller elements of $K d(\mathrm{~s})$ and $K o(\mathrm{~s})$ are obtained as shown in (44) and (45), respectively. The non-zero elements of $K d(\mathrm{~s})$ directly have PI structure, while those of $K o(\mathrm{~s})$ are a filtered derivative action plus a time delay. 


$$
\begin{aligned}
& k d_{i j}(s)=\frac{k_{j}}{k_{j i}} \frac{\left(T_{j i} s+1\right)}{s}=K_{P i j}+\frac{K_{I i j}}{s} \quad \forall i ; \quad j=p_{i}(44) \\
& k o_{i j}(s)=-\frac{k_{i j}}{k_{i}} \frac{s}{\left(T_{i j} s+1\right)} e^{-s\left(\theta_{i j}-\theta_{i}\right)}=\frac{K_{D i j} s}{T_{F i j} s+1} e^{-\theta\left(k_{i j}\right) s} \quad \forall i, j ; / i \neq p_{j}
\end{aligned}
$$

From (44) and (45), it can be found that the controller parameters are given by (46).

$$
\begin{aligned}
& K_{P i j}=T_{j i} \frac{k_{j}}{k_{j i}} \quad K_{I i j}=\frac{k_{j}}{k_{j i}} \quad \forall i ; \quad j=p_{i} \\
& K_{D i j}=-\frac{k_{i j}}{k_{i}} \quad T_{F i j}=T_{i j} \quad \theta\left(k o_{i j}\right)=\theta_{i j}-\theta_{i} \quad \forall i, j ; / i \neq p_{j}
\end{aligned}
$$

If the process elements can be fittingly approximated by FOPTD systems, authors propose to use the simple expressions given in (46) to calculate the controller elements of $K d(\mathrm{~s})$ and $K o(s)$ after determining the gain parameters $k_{i}$. However, when the process elements consist of pure integrators, RHP zeros, high relative degrees and so on, this approximation can work improperly. In this case, the controller elements should be reduced into more complex structures such as PID structure for $K d(\mathrm{~s})$ elements or lead-lag compensators with time delay for $K o(\mathrm{~s})$ elements.

\subsection{Practical considerations}

From an implementation point of view, it is important to consider how to solve practical problems such as wind-up, which can cause the controller to perform poorly in the presence of control signal constraints. When no extra dynamics $N(\mathrm{~s})$ is needed to guarantee the configuration realizability in the proposed method, the windup problem can be directly solved if the non-zero elements of $K d(\mathrm{~s})$ already have implemented some anti-windup mechanism. When they have PID structure, the simple anti-windup scheme in Figure 5 can be used. This scheme, which is

used for monovariable PID controllers, is based on back-calculations. ${ }^{32}$ It uses an input constraint model inside the controller. When the process input is saturated resulting in a different value than the PID output, the controller works in tracking mode following the saturated signal. 


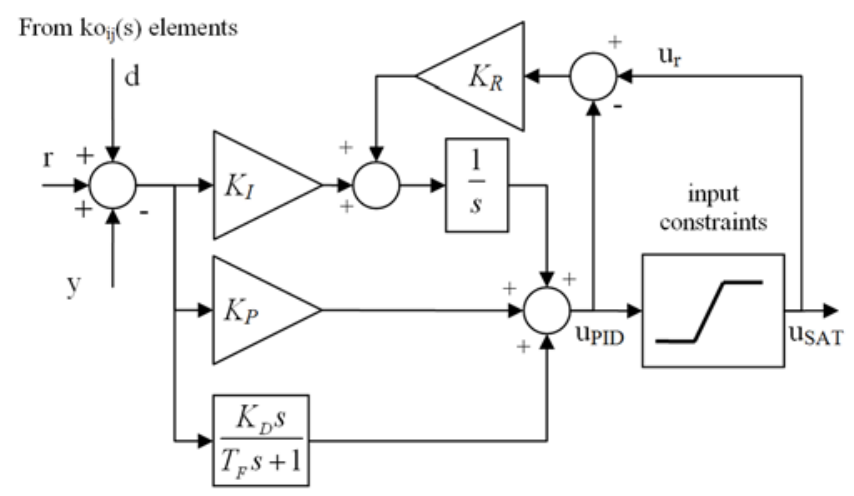

Figure 5. Anti-windup scheme for PID controllers in the proposed methodology.

According to the scheme of the proposed method in Figure 2, the control signals are fed back to the controller input and added to the error signals to decouple the system. When some control signal is saturated, this value is, in fact, the value which is fed back through the corresponding elements of $K o(s)$ toward the corresponding error signals in order to remain the other loops decoupled. This value is depicted by $d$ in Figure 5. Therefore, when saturations arise, there is an error conditioning ${ }^{29}$ which modifies the error vector in such a way that the control signals remain into allowable values. In this multivariable case, it is possible to use this simple monovariable scheme due to the structure of the centralized inverted decoupling control. In the conventional scheme of Figure 1b, it is more difficult to implement an anti-windup strategy assuring the decoupling performance.

On the other hand, bumpless transfer between operation modes (manual and automatic) can be easily achieved if the non-zero elements of $K d(\mathrm{~s})$ have implemented some monovariable strategy to assure this feature. Some mechanisms are based on the tracking mode of the final controller output. $^{32}$

In addition and similar to inverted decoupling, ${ }^{25}$ when the controller outputs are used as cascade setpoints to lower level controllers, each decoupled control loop is immune to abnormalities (e.g., a valve at a limit or a secondary controller in manual) in the secondary of the 
other loops. To obtain this decoupling performance, it is necessary to measure the output signals of the secondary loops and to feed back them through the elements of $K o(s)$.

\section{Examples}

In this section, two simulation processes are considered to demonstrate the proposed methodology, and its effectiveness is also verified in a real quadruple tank plant. These processes do not have multivariable RHP zeros, and therefore, the proposed method can be applied. More simulation examples of size $2 \times 2$ can be found in previous works. ${ }^{26,27}$

\subsection{Example 1: Vinante-Luyben distillation column}

The Vinante-Luyben distillation column ${ }^{8}$ is a multivariable system with important delays and it is described by the transfer matrix (47). Due to time delays, no configuration is initially realizable. It is necessary to insert an extra delay of $0.7 \mathrm{~min}$ in the second input, and therefore, the elements of $N(\mathrm{~s})$ are given by $n_{11}(\mathrm{~s})=1$ and $n_{22}(\mathrm{~s})=e^{-0.7 \mathrm{~s}}$. Then, the method is applied to (48). According to the conditions of Section 3, configuration 1-2 must be chosen for realizability.

$$
\begin{gathered}
G_{V}(\mathrm{~s})=\left(\begin{array}{cc}
\frac{-2.2 e^{-s}}{7 s+1} & \frac{1.3 e^{-0.3 s}}{7 s+1} \\
\frac{-2.8 e^{-1.8 s}}{9.5 s+1} & \frac{4.3 e^{-0.35 s}}{9.2 s+1}
\end{array}\right) \\
G_{V}^{N}(\mathrm{~s})=\left(\begin{array}{cc}
\frac{-2.2 e^{-s}}{7 s+1} & \frac{1.3 e^{-s}}{7 s+1} \\
\frac{-2.8 e^{-1.8 s}}{9.5 s+1} & \frac{4.3 e^{-1.05 s}}{9.2 s+1}
\end{array}\right)
\end{gathered}
$$

A gain margin of 3 and phase margin of $60^{\circ}$ are chosen as specifications in both loops. The gains $k_{1}$ and $k_{2}$ are obtained from (25) resulting the following open loop processes:

$$
L(s)=\left(\begin{array}{cc}
\frac{0.524}{\mathrm{~s}} e^{-s} & 0 \\
0 & \frac{0.499}{\mathrm{~s}} e^{-1.05 s}
\end{array}\right) .
$$


In addition, as the process elements are given by FOPTD systems, the expressions in (46) are used to calculate directly the parameters of the controller elements. The following controllers in (50) are achieved. They have directly structure of PI control or filtered derivative action plus time delay.

$$
\begin{gathered}
K d(s)=\left(\begin{array}{cc}
-1.666-\frac{0.238}{s} & 0 \\
0 & 1.067+\frac{0.116}{s}
\end{array}\right) \\
K o(s)=\left(\begin{array}{cc}
0 & \frac{-2.483 s}{7 s+1} \\
\frac{5.615 s}{9.5 s+1} e^{-0.75 s} & 0
\end{array}\right)
\end{gathered}
$$

Figure 6 shows the closed loop system response of the proposed methodology in comparison with that of the normalized decoupling, ${ }^{8}$ which is designed with the same performance specifications. A decentralized PI control ${ }^{33}$ is also shown. There is a unit step change in the first reference at $t=1 \mathrm{~min}$, and at $t=40 \mathrm{~min}$, in the second one. At $t=70 \mathrm{~min}$, there is a $0.5 \mathrm{step}$ in both process inputs as load disturbances. The IAE indices are collected in Table 2. 

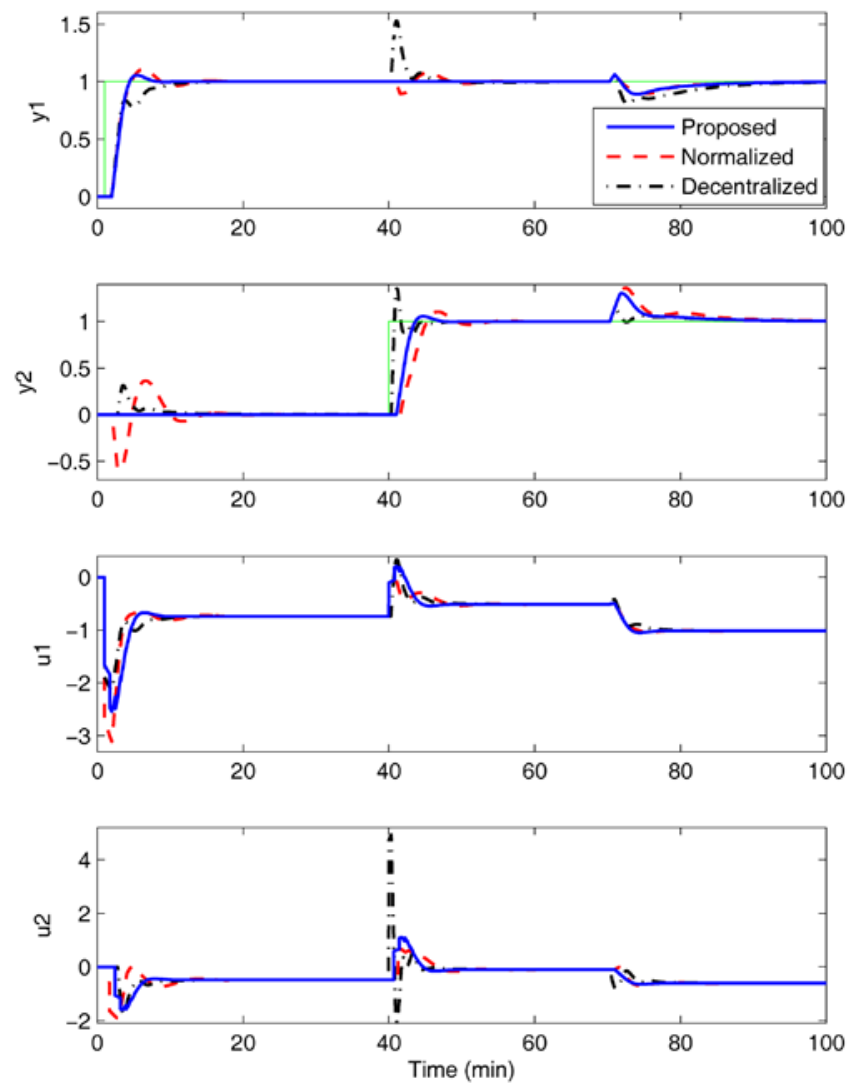

Figure 6. Outputs and control signals of the step response of Example 1.

In the first loop, the proposed control achieves the best response with the smallest IAE values. The decentralized control obtains the best performance in the second loop, with fast reference tracking and disturbance rejection. However, this response is obtained at expense of an important overshoot and very large control signals. The proposed control achieves perfect decoupling, while the others present important interactions. The response of the proposed control is better than that of the normalized decoupling. In addition, another advantage of the proposed methodology over normalized decoupling is its direct method of carrying out the design. In the normalized decoupling design, the procedure is slightly more complex with the calculation of the normalized gain matrix, the RGA, the RNGA and the RARTA.

Table 2. IAE values and robustness indices for each method in Example 1. 


\begin{tabular}{|c|c|c|c|c|c|c|c|}
\hline Method & IAE & Tracking & Interaction & Disturbance & Total & $\mu_{R S}$ & $\mu_{R P}$ \\
\hline \multirow{2}{*}{ Proposed } & loop 1 & 2.14 & $2 \cdot 10^{-4}$ & 0.94 & 3.08 & \multirow{2}{*}{0.28} & \multirow{2}{*}{1.04} \\
\hline & loop 2 & 2.25 & 0.001 & 1.47 & 3.72 & & \\
\hline \multirow{2}{*}{ Normalized } & loop 1 & 2.43 & 0.47 & 0.9 & 3.80 & \multirow{2}{*}{0.61} & \multirow{2}{*}{1.7} \\
\hline & loop 2 & 3.62 & 2.44 & 2.22 & 8.28 & & \\
\hline \multirow{2}{*}{$\begin{array}{c}\text { Decentralize } \\
\mathrm{d}\end{array}$} & loop 1 & 2.63 & 0.95 & 1.8 & 5.38 & \multirow{2}{*}{0.67} & \multirow{2}{*}{1.63} \\
\hline & loop 2 & 1.03 & 0.81 & 0.82 & 2.66 & & \\
\hline
\end{tabular}

To evaluate the robustness of the proposed controllers, a $\mu$-analysis is performed in the presence of diagonal multiplicative input uncertainty. To achieve robust stability, the necessary and sufficient condition ${ }^{31}$ is

$$
\mu_{\mathrm{RS}}=\mu\left[-\mathrm{W}_{\mathrm{I}}(\mathrm{s}) \mathrm{T}_{\mathrm{I}}(\mathrm{s})\right]<1 \quad \forall \omega
$$

where $\mu$ is the structured singular value (SSV) and $T_{I}(\mathrm{~s})$ (equal to $K(\mathrm{~s}) \cdot G(\mathrm{~s}) \cdot(I+K(\mathrm{~s}) \cdot G(\mathrm{~s}))^{-1}$ ) is the input complementary sensitivity function. $W_{I}(\mathrm{~s})$ and $W_{P}(\mathrm{~s})$ are the diagonal weights for uncertainty and performance, respectively. To evaluate whether the closed loop system will respect the desired performance even in the presence of diagonal multiplicative input uncertainty, the necessary and sufficient condition ${ }^{31}$ is

$$
\mu_{\mathrm{RP}}=\mu\left[\begin{array}{cc}
-W_{I}(s) T_{I}(s) & -W_{I}(s) K(s) S(s) \\
W_{P}(s) S(s) G(s) & W_{P}(s) S(s)
\end{array}\right]<1 \quad \forall \omega .
$$

In this example, the selected weights are

$$
\begin{aligned}
& W_{I}(\mathrm{~s})=W_{I}(\mathrm{~s})=\frac{(0.4 \mathrm{~s}+0.15)}{s+1} \cdot I \\
& W_{P}(\mathrm{~s})=W_{P}(\mathrm{~s})=\frac{(\mathrm{s} / 2.6+0.01)}{s} \cdot I
\end{aligned} \cdot
$$

The weight $w_{I}(\mathrm{~s})$ can be loosely interpreted as the process inputs increase by up to $40 \%$ uncertainty at high frequencies and by almost $15 \%$ uncertainty in the low frequency range. The performance weight $w_{P}(\mathrm{~s})$ specifies integral action and a maximum peak for $\bar{\sigma}(S)$ of $M_{S}=2.6$. Figure 7 shows the SSV for robust stability (RS) and robust performance (RP) for the different 
controllers under conditions (51) and (52). The RS is smaller than one for all frequencies, indicating that the systems will remain stable in spite of an uncertainty of $15 \%$ on each process input. The peak values are shown in Table 2. The proposed controller has the smallest value. The RP analysis shows that the proposed method is the only one that almost satisfies the RP condition (52) with a very little peak value of 1.04 around $1 \mathrm{rad} / \mathrm{min}$. For the other controllers, the performance will deteriorate around this frequency, where the peaks appear. These values are also collected in Table 2. The decentralized controller has a good RS and the best RP for low frequencies; however, it shows a bad RP for frequencies higher than $1 \mathrm{rad} / \mathrm{min}$.
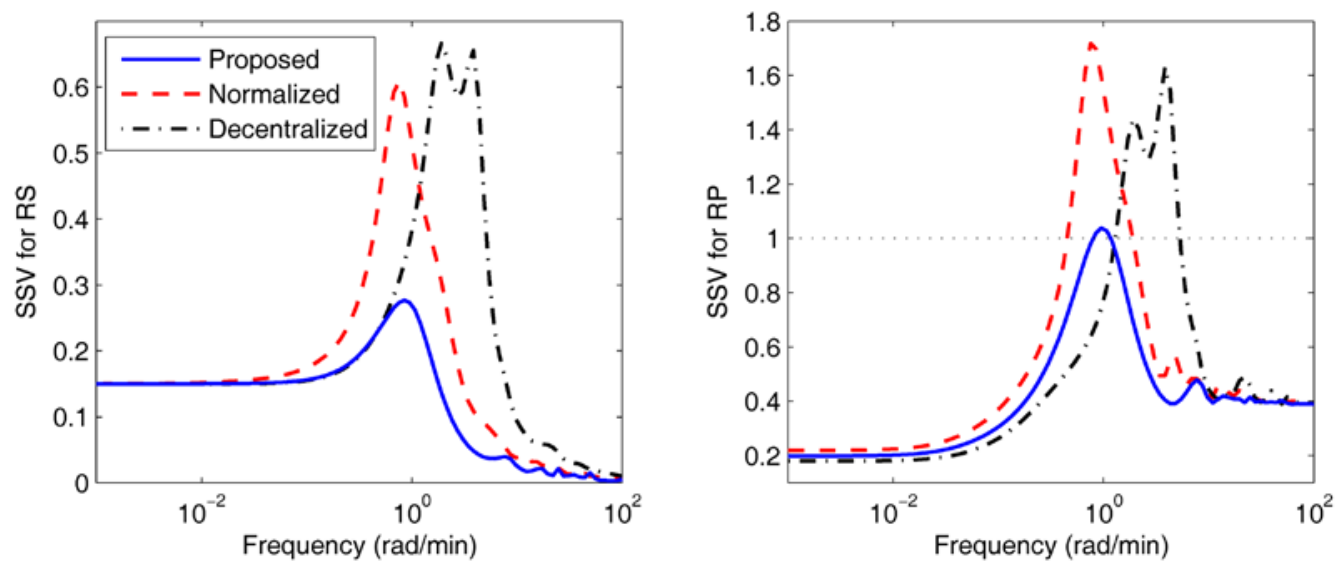

Figure 7. SSV for RS and RP in Example 1.

Next, a simulation is performed with input constraints in order to show the windup problem and test the anti-windup (AW) scheme of Figure 5. The process inputs are limited in the range of $[-0.7,0.7]$. At $t=1 \mathrm{~min}$, there is a unit step change in the first reference, and then, at $t=50 \mathrm{~min}$, there is a similar step change in the second one. The proposed controller is tested assuming two cases: without anti-windup mechanism and using the proposed anti-windup scheme. Figure 8 shows the simulation results. The response of the decentralized control is also obtained using the anti-windup mechanism of Figure 5 without the $d$ input signal. 
After the first reference change, the first control signal $u_{1}$ should be out of range to track this reference. However, its lower limit of $u_{1}$ is reached and the first reference cannot be achieved. In the first case, without anti-windup mechanism, this fact provokes windup in this signal and after the second reference change, at $t=50 \mathrm{~min}, u_{1}$ does not change until 20 min later. Consequently, the time to reach the new references is very late. In addition, after the first reference change, the second reference is also lost because the directionality of the control signal vector is modified after the lower limit of $u_{1}$ is reached and interactions arise.

This response is improved significantly over the implementation of the anti-windup scheme of Figure 5. Using this mechanism in the $K d(\mathrm{~s})$ elements, the first reference is not reached at the beginning; however, the second reference is not lost. When $u_{1}$ is limited, the control signal $u_{2}$ is automatically limited due to the inverted decoupling structure, in such a way that the second loop remains decoupled. Additionally, the windup effect has been avoided, the control signal $u_{1}$ reacts very quickly after the second reference step, and all references are reached sooner. The responses of the decentralized controller show some interaction and the settling time of the second loop is greater. 

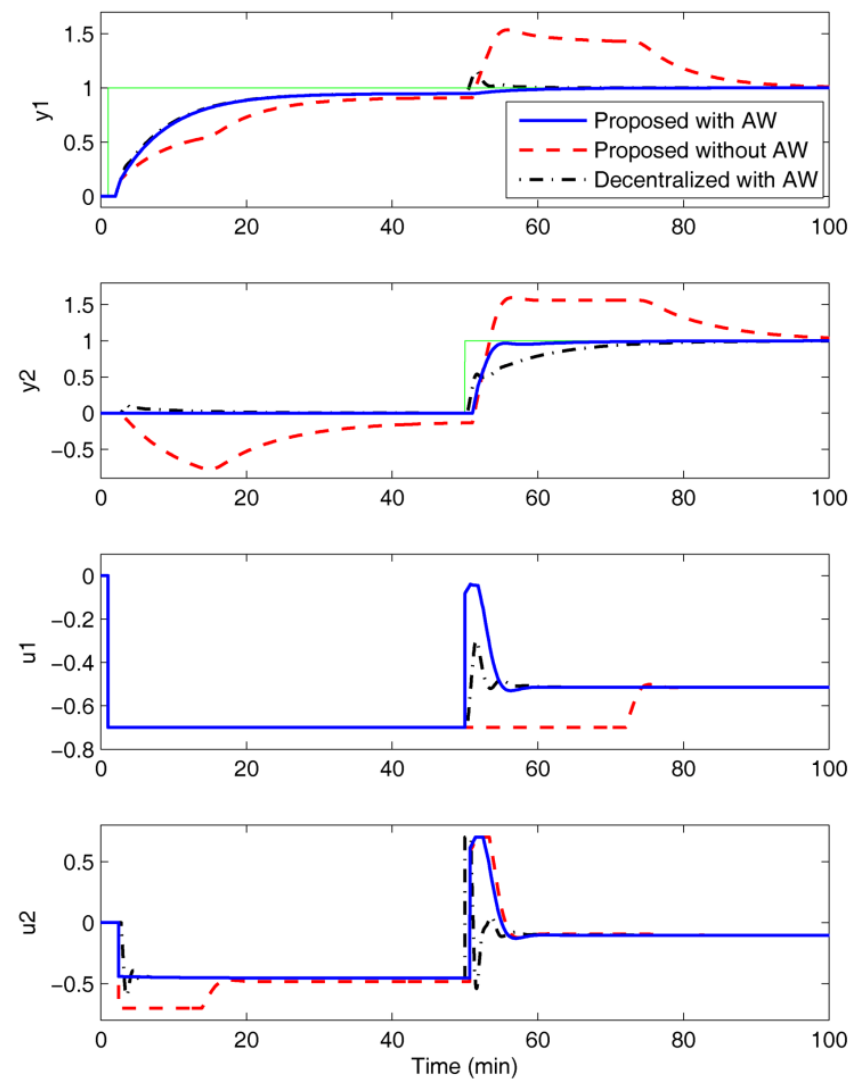

Figure 8. Outputs and control signals of the step response of Example 1 with input constraints.

\subsection{Example 2: $3 \times 3$ Tyreus distillation column}

This second system is a sidestream column separating a ternary mixture. ${ }^{34}$ Its transfer matrix is given by (54), where the controlled and manipulated variables are $y_{1}$ (toluene impurity in the distillate); $y_{2}$ (benzene impurity in the sidestream); $y_{3}$ (toluene impurity in the bottom); $u_{1}$ (reflux ratio); $u_{2}$ (sidestream flow rate); $u_{3}$ (reboil duty). Due to time delays, there are no realizable configurations for this process. Thus, it is necessary to include an additional block $N(\mathrm{~s})$ with delays. To achieve realizability by adding the minimum quantity of delays, the only choice is configuration 1-2-3 with $n_{11}(\mathrm{~s})=e^{-0.09 \mathrm{~s}}, n_{22}(\mathrm{~s})=1$ and $n_{33}(\mathrm{~s})=e^{-0.26 \mathrm{~s}}$. The new augmented process $G(\mathrm{~s}) \cdot N(\mathrm{~s})$ to be decoupled is given by (55). 


$$
\begin{aligned}
G_{T}(s) & =\left(\begin{array}{ccc}
\frac{1.986 e^{-0.71 s}}{66.7 s+1} & \frac{-5.24 e^{-60 s}}{400 s+1} & \frac{-5.984 e^{-2.24 s}}{14.29 s+1} \\
\frac{-0.0204 e^{-0.59 s}}{(7.14 s+1)^{2}} & \frac{0.33 e^{-0.68 s}}{(2.38 s+1)^{2}} & \frac{-2.38 e^{-0.42 s}}{(1.43 s+1)^{2}} \\
\frac{-0.374 e^{-7.75 s}}{22.22 s+1} & \frac{11.3 e^{-3.79 s}}{(21.74 s+1)^{2}} & \frac{9.811 e^{-1.59 s}}{11.36 s+1}
\end{array}\right) \\
G_{T}^{N}(s) & =\left(\begin{array}{ccc}
\frac{1.986 e^{-0.8 s}}{66.7 s+1} & \frac{-5.24 e^{-60 s}}{400 s+1} & \frac{-5.984 e^{-2.5 s}}{14.29 s+1} \\
\frac{-0.0204 e^{-0.68 s}}{(7.14 s+1)^{2}} & \frac{0.33 e^{-0.68 s}}{(2.38 s+1)^{2}} & \frac{-2.38 e^{-0.68 s}}{(1.43 s+1)^{2}} \\
\frac{-0.374 e^{-7.84 s}}{22.22 s+1} & \frac{11.3 e^{-3.79 s}}{(21.74 s+1)^{2}} & \frac{9.811 e^{-1.85 s}}{11.36 s+1}
\end{array}\right)
\end{aligned}
$$

The process elements of the first and third rows are FOPTD systems, and consequently, the corresponding open loop processes $l_{1}(\mathrm{~s})$ and $l_{3}(\mathrm{~s})$ can be chosen according to case 1 of Table 1 . On the other hand, the process elements of the second row have relative degree equal to two, and therefore, the associated open loop process $I_{2}(\mathrm{~s})$ must be defined according to the second case of Table 1. A gain margin of 10 is chosen as specification in the three loops. The gains $k_{1}$ and $k_{3}$ are calculated by means of (25). In the second open loop process $l_{2}(\mathrm{~s})$, the time constant $\lambda$ of the extra pole is obtained from (28) after specifying $\omega_{c p}=0.63 \mathrm{rad} / \mathrm{min}$. Then, the gain $k_{2}$ is calculated according to (27). The resulting open loop processes are given by:

$$
L(s)=\operatorname{diag}\left(\frac{0.196}{\mathrm{~s}} e^{-0.8 s}, \frac{0.152}{\mathrm{~s} \cdot(3.476 \mathrm{~s}+1)} e^{-0.68 s}, \frac{0.085}{\mathrm{~s}} e^{-1.85 s}\right)
$$

After selecting the configuration 1-2-3 and defining the desired open loop transfer functions, the controller elements are obtained according to (12) without approximations, as follows:

$$
K d(s)=\left(\begin{array}{ccc}
\frac{6.59 \cdot(s+0.015)}{s} & 0 & 0 \\
0 & \frac{0.749 \cdot(s+0.42)^{2}}{s \cdot(s+0.288)} & 0 \\
0 & 0 & \frac{0.098 \cdot(s+0.088)}{s}
\end{array}\right) \text {, }
$$




$$
K o(s)=\left(\begin{array}{ccc}
0 & \frac{0.067 \cdot s \cdot e^{-59 s}}{s+0.0025} & \frac{2.13 \cdot s \cdot e^{-1.7 s}}{s+0.07} \\
\frac{9.17 \cdot 10^{-3} s \cdot(s+0.288)}{(s+0.14)^{2}} & 0 & \frac{26.7 \cdot s \cdot(s+0.29)}{(s+0.7)^{2}} \\
\frac{0.198 \cdot s \cdot e^{-6 s}}{s+0.045} & \frac{-0.28 \cdot s \cdot e^{-1.9 s}}{(s+0.046)^{2}} & 0
\end{array}\right) .
$$

The closed loop system responses (outputs and control signals) are shown in Figure 9. There are unit step changes at $t=5 \mathrm{~min}$ in the first reference, at $t=200 \mathrm{~min}$, in the second one, and $t=$ 400 min, in the third one. For comparison, other control techniques are also shown in this figure: the high order centralized control of Wang ${ }^{7}$ and the decentralized PID controller of He. ${ }^{35}$
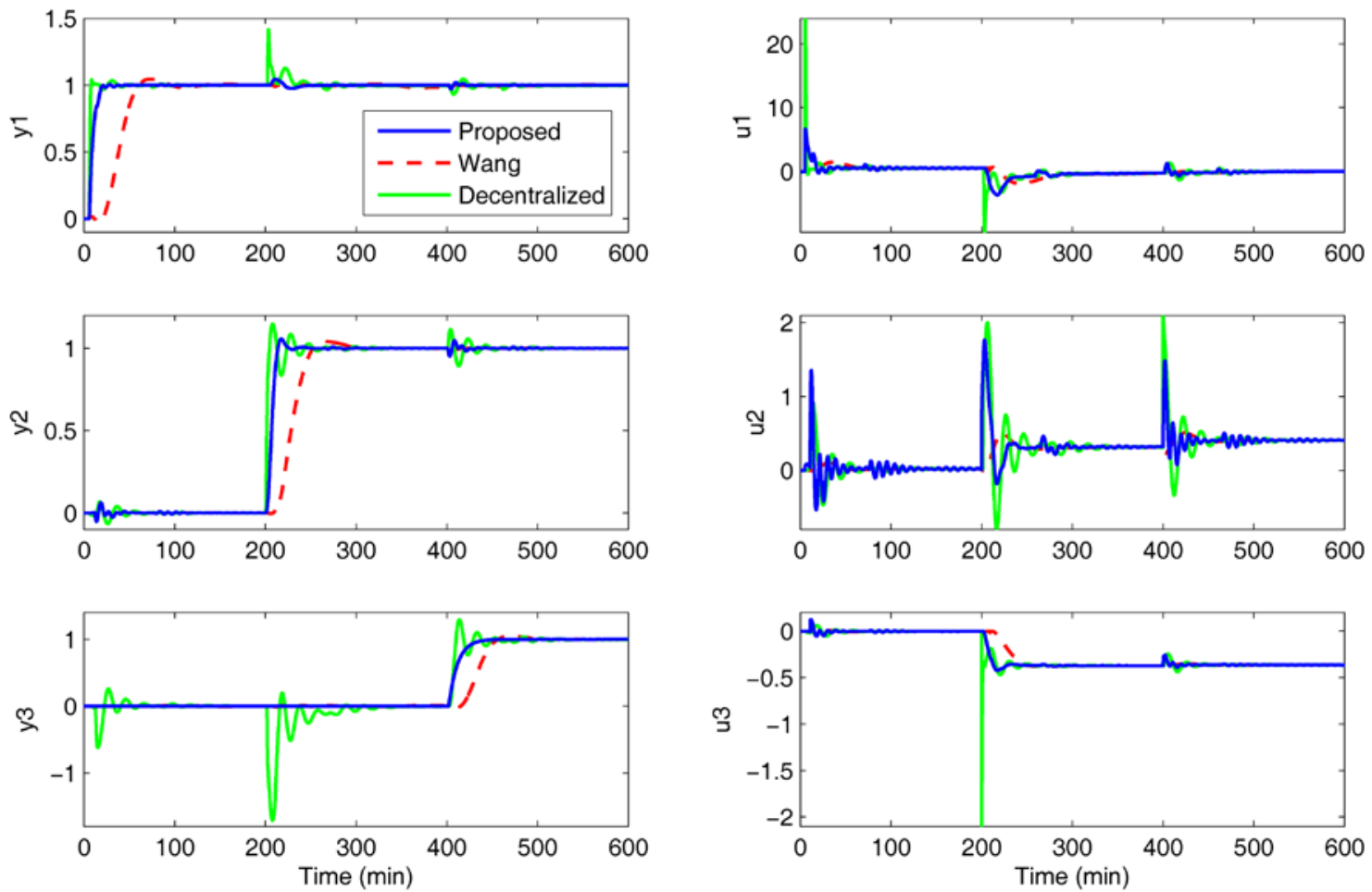

Figure 9. Outputs and control signals of the step response of Example 2.

The proposed design and Wang's control obtain almost perfect decoupling performance, while the decentralized control presents important interactions in the first and third outputs and very large control signals. Although the control signals of the proposed controller are rather 
oscillatory, the resulting closed loop responses have smaller settling times than those of obtained with the Wang's control. The IAE values of the three methods are listed in Table 3 as performance indices. Although the smallest IAE values for tracking are achieved by the decentralized controller, the proposed control obtains the smallest total ones.

Table 3. IAE values and robustness indices for each method in Example 2.

\begin{tabular}{c|cccc|cc}
\hline Method & IAE & Tracking & Interaction & Total & $\boldsymbol{\mu}_{\boldsymbol{R} S}$ & $\boldsymbol{\mu}_{\boldsymbol{R} P}$ \\
\hline \multirow{3}{*}{ Proposed } & loop 1 & 5.3 & 1.2 & 6.5 & & \\
& loop 2 & 7.5 & 1.5 & 9 & 0.45 & 1.05 \\
& loop 3 & 11.9 & 0.1 & 12 & & \\
\cline { 2 - 5 } Wang & loop 1 & 35.6 & 2.2 & 37.8 & & \\
& loop 2 & 30.7 & 0.3 & 31 & 0.15 & 1.09 \\
Decentralize & loop 3 & 35.1 & 2.4 & 37.5 & & \\
d & loop 1 & 2.5 & 5.7 & 8.2 & & \\
& loop 2 & 5.5 & 3.5 & 9 & 0.96 & 3.08 \\
& loop 3 & 13 & 36 & 49 & & \\
\hline
\end{tabular}

To investigate the robustness of the three controllers, a $\mu$-analysis similar to the previous example is performed. The selected weights are given by (59). Figure 10 shows the SSV for RS and RP for the different controllers. The RS is satisfied by all of them; however, the smallest values are obtained by Wang's controller. The other peak values are collected in Table 3. The RP condition (52) is almost satisfied by the proposed control and Wang's controller with peak values lower than 1.1. The decentralized controller shows the worst robust performance at frequencies above $0.1 \mathrm{rad} / \mathrm{min}$, due to the strong interactions.

$$
\begin{aligned}
& W_{I}(\mathrm{~s})=W_{I}(\mathrm{~s})=\frac{(0.7 \mathrm{~s}+0.15)}{s+1} \cdot I \\
& W_{P}(\mathrm{~s})=W_{P}(\mathrm{~s})=\frac{(\mathrm{s} / 2.7+0.0125)}{s} \cdot I
\end{aligned}
$$



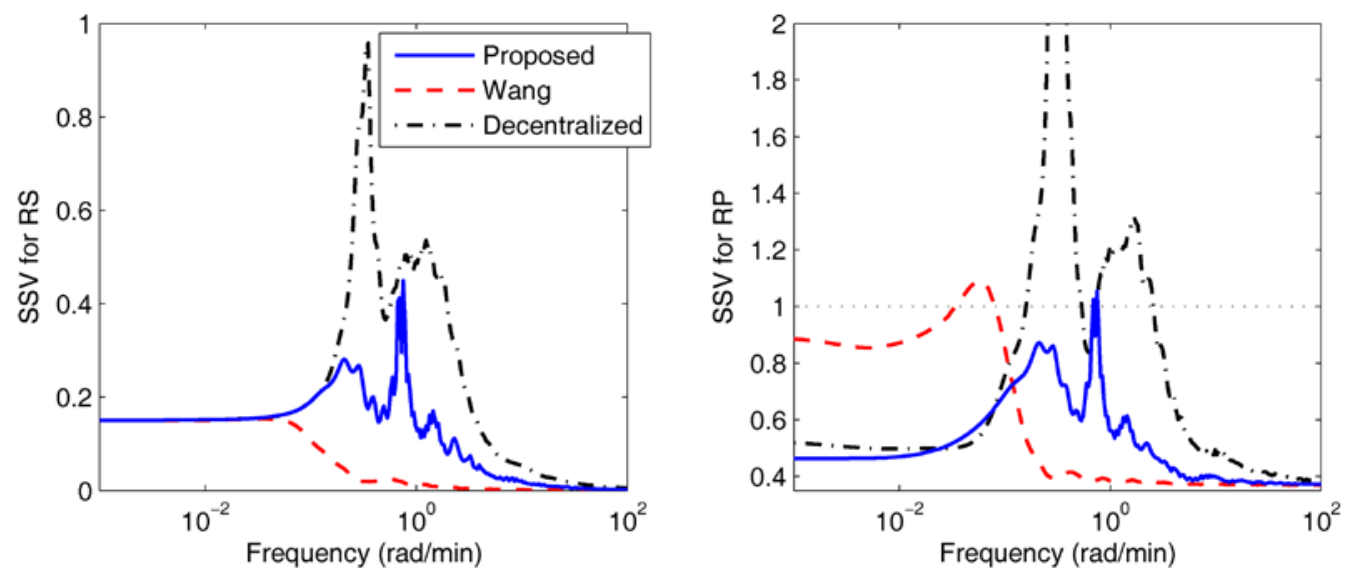

Figure 10. SSV for RS and RP in Example 2.

\subsection{Example 3: Experimental quadruple tank system}

This experimental process is a quadruple tank plant ${ }^{36}$ in the lab of the Computer Science Department of the University of Cordoba. The outputs are the level of the lower tanks, which are between 0 and $35 \mathrm{~cm}$; the inputs are the flow references of the secondary control loops that regulate the operation of the pumps, which are between 0 and $200 \mathrm{~cm}^{3} / \mathrm{s}$. The plant was configured in order to show interaction problems without having multivariable RHP zeros. The process was identified around the operation point $h=\left[\begin{array}{ll}20 & 20\end{array}\right] \mathrm{cm}$ and $u=\left[\begin{array}{lll}138 & 135\end{array}\right] \mathrm{cm} 3 / \mathrm{s}$. the resultant model is given by (60) and it has a relative gain array of 2.29.

$$
G_{Q}(s)=\left(\begin{array}{cc}
\frac{0.3284}{184.5 s+1} & \frac{0.2454}{(184.5 s+1)(535.1 s+1)} \\
\frac{0.2457}{(185 s+1)(503.2 s+1)} & \frac{0.3378}{185 s+1}
\end{array}\right)
$$

Because of the relative degrees, configuration 1-2 must be chosen for realizability without adding extra dynamics. In addition, the two open loop transfer functions $l_{1}(\mathrm{~s})$ and $l_{2}(\mathrm{~s})$ can be defined according to the case 1 of Table 1, and since there are no time delays, the closed loop transfer functions $h_{1}$ (s) and $h_{2}$ (s) are given by (24). Therefore, a closed loop time constant of $300 \mathrm{~s}$ is chosen as specifications in both loops. Consequently, the parameters $k_{1}$ and $k_{2}$ are 
calculated as the inverse of this time constant. As a result, the desired open loop transfer functions are defined by $l_{1}(\mathrm{~s})=l_{2}(\mathrm{~s})=1 /(300 \cdot s)$.

After specifying the functions $l_{i}(\mathrm{~s})$, the controller elements are obtained from (9) as follows:

$$
\begin{aligned}
& k d_{11}(\mathrm{~s})=1.873+\frac{1}{98.52 \mathrm{~s}} \quad k o_{12}(\mathrm{~s})=\frac{-73.62 \mathrm{~s}}{(184.5 \mathrm{~s}+1)(535.1 \mathrm{~s}+1)} . \\
& k o_{21}(\mathrm{~s})=\frac{-73.71 \mathrm{~s}}{(185 \mathrm{~s}+1)(503.2 \mathrm{~s}+1)} \quad k d_{22}(\mathrm{~s})=1.825+\frac{1}{101.34 \mathrm{~s}}
\end{aligned}
$$

Figure 11 shows the resultant response of the proposed control for a step of $2 \mathrm{~cm}$ in both references. For comparison, the response of a multi-loop PI controller given by (62) is also shown. This decentralized controller is tuned according to an iterative method $^{2}$ using a phase margin of $90^{\circ}$ and an infinite gain margin as specifications in both loops.
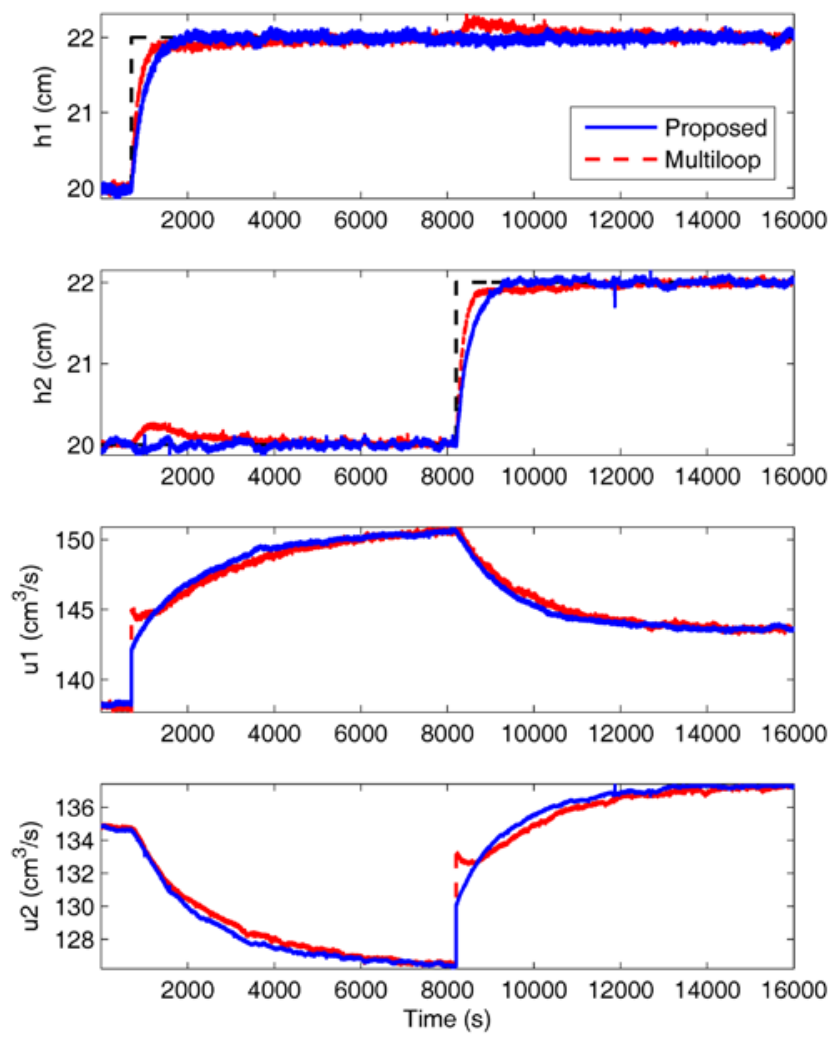

Figure 11. Outputs and control signals of the step response of the quadruple tank system. 


$$
k_{11}(s)=3.44+\frac{1}{54.323 s} \quad k_{22}(s)=3.35+\frac{1}{55.93 s}
$$

The proposed control obtains a better response with a very good decoupling performance and a smaller settling time in both loops. The multi-loop PI controller reaches the references later, and the rejection of the interactions is very slow. The IAE values for both methods are listed in Table 4 as performance indices. The proposed control obtains the smallest total IAE values.

Table 4. IAE values for each method in Example 3.

\begin{tabular}{c|cccc}
\hline Method & IAE & Tracking & Interaction & Total \\
\hline \multirow{2}{*}{ Proposed } & loop 1 & 732 & 307 & 1039 \\
& loop 2 & 773 & 227 & 1000 \\
\cline { 2 - 5 } Multiloop & loop 1 & 716 & 451 & 1167 \\
& loop 2 & 733 & 422 & 1155 \\
\hline
\end{tabular}

\section{Conclusions}

A new methodology of multivariable centralized control based on the structure of inverted decoupling is developed in this work. The problem is approached from a compact matricial formulation obtaining generalized expressions for $n \times n$ processes. From them, it is demonstrated that the controller elements are very simple and they can be calculated very easily in comparison with other methods based on the conventional centralized scheme in which complicated elements are often achieved and approximations are usually necessary. Furthermore, it is found that the complexity of the controller elements is independent of the system size. This is a great advantage over the conventional scheme. Additionally, the structure of the proposed centralized scheme allows for dealing with other practical problems, such as anti-windup or bumpless transfer, in the same way as in the monovariable case. These practical issues are not solved so easily with the conventional centralized scheme. Therefore, it is concluded that the proposed methodology has 
important advantages that makes it very easy to apply when the process does not have multivariable RHP zeros.

The possible configurations and the realizability conditions for applying the method were discussed. In addition, three common cases to select performance specifications from simple open loop transfer functions are explained. As a particular case, the controller parameter expressions when all of the process elements are given by FOPTD systems were obtained. In this case, the controller elements have PI structure or filtered derivative action plus a time delay. The method has been illustrated with two simulation examples. Comparisons with other methods have demonstrated that the proposed methodology achieves similar or better performance. In addition, an experimental quadruple tank process was used to verify the effectiveness of this method.

\section{Acknowledgments}

This work was supported by the Autonomous Government of Andalusia (Spain), under the Excellence Project P10-TEP-6056. This support is very gratefully acknowledged.

\section{References}

(1) Shinskey, F. G., Process Control Systems: Application, Design and Adjustment. 2 ed.; McGraw-Hill: New York, 1979.

(2) Vázquez, F.; Morilla, F.; Dormido, S., An iterative method for tuning decentralized PID controllers. In Proceedings of the 14th IFAC World Congress, Beijing, China, 1999; pp 491-496.

(3) Huang, H. P.; Jeng, J. C.; Chiang, C. H.; Pan, W., A direct method for multi-loop PI/PID controller design. J. Process Control 2003, 13, 769-786.

(4) Lee, M.; Lee, K.; Kim, C.; Lee, J., Analytical design of multiloop PID controllers for desired closed-loop responses. AIChE J. 2004, 50, 1631-1635.

(5) Xiong, Q.; Cai, W.-J., Effective transfer function method for decentralized control system design of multiinput multi-output processes. J. Process Control 2006, 16, 773-784.

(6) Chien, I. L.; Huang, H. P.; Yang, J. C., A simple multiloop tuning method for PID controllers with no proportional kick. Ind. Eng. Chem. Res. 1999, 38, 1456-1468.

(7) Wang, Q. G., Decoupling Control. Springer-Verlag: Berlin-Heidelberg, 2003.

(8) Cai, W. J.; Ni, W.; He, M. J.; Ni, C. Y., Normalized decoupling - a new approach for MIMO process control system design. Ind. Eng. Chem. Res. 2008, 47, 7347-7356.

(9) Garrido, J.; Vázquez, F.; Morilla, F., An extended approach of inverted decoupling. J. Process Control 2011, 21, 55-68. 
(10) Garrido, J.; Vázquez, F.; Morilla, F., Centralized multivariable control by simplified decoupling. J. Process Control 2012, 22, 1044-1066.

(11) Jevtovic, B. T.; Matausek, M. R., PID controller design of TITO system based on ideal decoupler. J. Process Control 2010, 20, 869-876.

(12) Nordfeldt, P.; Hägglund, T., Decoupler and PID controller design of TITO systems. J. Process Control 2006, 16, 923-936.

(13) Rajapandiyan, C.; Chidambaram, M., Controller design for MIMO processes based on simple decoupled equivalent transfer functions and simplified decoupler. Ind. Eng. Chem. Res. 2012, 51, 12398-12410.

(14) Shen, Y.; Sun, Y.; Li, S., Adjoint transfer matrix based decoupling control for multivariable processes. Ind. Eng. Chem. Res. 2012, 51, 16419-16426.

(15) Wade, H. L., Inverted decoupling: A neglected technique. ISA Trans. 1997, 36, 3-10.

(16) Waller, M.; Waller, J. B.; Waller, K. V., Decoupling Revisited. Ind. Eng. Chem. Res. 2003, 42, 4575-4577.

(17) Lieslehto, J., MIMO controller design using SISO controller design methods. In Proceeding of the 13th IFAC World Congress, San Francisco, USA, 1996; pp 169-173.

(18) Wang, Q. G.; Zou, B.; Lee, T. H.; Bi, Q., Auto-tuning of multivariable PID controllers from decentralized relay feedback. Automatica 1997, 33, 319-330.

(19) Wang, Q. G.; Zhang, Y.; Chiu, M. S., Decoupling internal model control for multivariable systems with multiple time delays. Chem. Eng. Sci. 2002, 57, 115-124.

(20) Wang, Q. G.; Zhang, Y.; Chiu, M. S., Non-interacting control design for multivariable industrial processes. J. Process Control 2003, 13, 253-265.

(21) Xiong, Q.; Cai, W. J.; He, M. J., Equivalent transfer function method for PI/PID controller design of MIMO processes. J. Process Control 2007, 17, 665-673.

(22) Morilla, F.; Vázquez, F.; Garrido, J., Centralized PID control by decoupling for TITO processes. In Proceedings of the 13th IEEE International Conference on Emerging Technologies and Factory Automation, Hamburg, Germany, 2008; pp 1318-1325.

(23) Liu, T.; Zhang, W.; Gao, F., Analytical decoupling control strategy using a unity feedback control structure for MIMO processes with time delays. J. Process Control 2007, 17, 173-186.

(24) Zhang, W.; Chen, L.; Ou, L., Algebraic Solution to H2 Control Problems. II. The Multivariable Decoupling Case. Ind. Eng. Chem. Res. 2006, 45, 7163-7176.

(25) Garrido, J.; Vázquez, F.; Morilla, F.; Hägglund, T., Practical advantages of inverted decoupling. Proc. Inst. Mech. Eng. I J. Syst. Control Eng. 2011, 225, 977-992.

(26) Garrido, J.; Vázquez, F.; Morilla, F., Centralized Inverted Decoupling for TITO Processes. In Proceedings of the 15th IEEE International Conference on Emerging Technologies and Factory Automation, Bilbao, Spain, 2010.

(27) Garrido, J.; Vázquez, F.; Morilla, F., Multivariable PID Control by Inverted Decoupling: Application to the Benchmark PID 2012. In IFAC Conference on Advances in PID Control PID'12, Brescia, Italy, 2012.

(28) Rivera, D. E.; Morari, M.; Skogestad, S., Internal model control. 4. PID controller design. Ind. Eng. Chem. Process Des. Dev. 1986, 25, 252-265.

(29) Goodwin, G. C.; Graebe, S. F.; Salgado, M. E., Control System Design. Prentice-Hall: New Jersey, USA, 2001.

(30) Pintelon, R.; Guillaume, P.; Rolain, Y.; Schoukens, J.; Van hamme, H., Parametric identification of transfer functions in the frequency domain - A survey. IEEE Trans. Autom. Control 1994, 39, 2245-2260.

(31) Skogestad, S.; Postlethwaite, I., Multivariable Feedback Control: Analysis and Design. 2 ed.; John Wiley \& sons: Chichester, England, 2005.

(32) Áström, K.; Hägglund, T., Advanced PID control. ISA-The Instrumentation, Systems, and Automation Society: Research Triangle Park, North Carolina, 2006.

(33) Luan Vu, T. N.; Lee, M., Independent design of multi-loop PI/PID controllers for interacting multivariable processes. J. Process Control 2010, 20, 922-933.

(34) Tyreus, B. D., Multivariable control system design for an industrial distillation column. Ind. Eng. Chem. Process Des. Dev. 1979, 18, 177-182.

(35) He, M.-J.; Cai, W.-J.; Wu, B.-F., Design of decentralized IMC-PID controller based on dRI analysis. AIChE J. 2006, 52, 3852-3863.

(36) Johansson, K. H., The quadruple-tank process: A multivariable laboratory process with an adjustable zero. IEEE Trans Control Syst Technol. 2000, 8, 456-465. 